\title{
Deep Neck Inflammation: Probable Kawasaki Disease in Korean Children
}

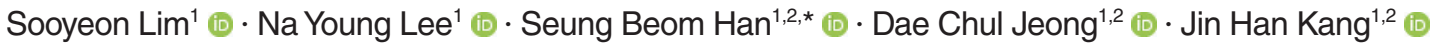 \\ ${ }^{I}$ Department of Pediatrics and ${ }^{2}$ The Vaccine Bio Research Institute, College of Medicine, The Catholic University of Korea, Seoul, Korea
}

Objectives. Deep neck infections (DNIs) can cause life-threatening complications, and prompt diagnosis and management are necessary. Kawasaki disease (KD) may be accompanied by deep neck inflammation; making it difficult to distinguish from DNIs. This study was performed to evaluate clinical features and outcomes of children with parapharyngeal and retropharyngeal inflammation.

Methods. Medical records of the children diagnosed with parapharyngeal and retropharyngeal cellulitis or abscess using cervical computed tomography (CT) between 2013 and 2017 were retrospectively reviewed.

Results. A total of 47 children were diagnosed with parapharyngeal and retropharyngeal inflammation. Eleven (23.4\%) of them were eventually diagnosed with $\mathrm{KD}$, and $36(76.6 \%)$ were diagnosed with DNIs. There were no significantly different clinical and laboratory characteristics on admission between children diagnosed with KD and DNIs; however, significantly more children with KD were febrile for $\geq 3$ days after admission compared to those with DNIs $(P=0.009)$. Deep neck abscesses on CT were observed in 16 children with DNIs $(44.4 \%)$ and in no child with KD $(P=0.009)$. Among the 36 children with DNIs, $30(83.3 \%)$ were cured with antibiotic therapy only.

Conclusion. A quarter of children presenting with deep neck inflammation were diagnosed with KD. KD should be considered in children showing deep neck inflammation unresponsive to empirical antibiotic therapy after 3 days, especially in those presenting with deep neck cellulitis rather than deep neck abscess.

Keywords. Retropharyngeal Abscess; Kawasaki Disease; Child; Korea

\section{INTRODUCTION}

Deep neck infections (DNIs) occur in potential deep neck spaces formed by deep cervical fascia including parapharyngeal, retropharyngeal, peritonsillar, submandibular, parotid, buccal, and carotid spaces [1]. DNIs can result in life-threatening complications, including airway obstruction and those caused by local dissemination: mediastinitis, necrotizing fasciitis, aspiration

- Received June 21, 2019

Revised August 20, 2019

Accepted August 23, 2019

- Corresponding author: Seung Beom Han

Department of Pediatrics, Daejeon St. Mary's Hospital, College of Medicine,

The Catholic University of Korea, 64 Daeheung-ro, Jung-gu, Daejeon 34943, Korea

Tel: +82-42-220-9103, Fax: +82-42-221-2925

E-mail: beomsid@catholic.ac.kr

*The author is now working at Department of Pediatrics, Daejeon St. Mary's Hospital, College of Medicine, The Catholic University of Korea, Daejeon, Korea. pneumonia, jugular vein thrombophlebitis and distant embolism, carotid artery aneurysm and rupture, and cranial nerve palsies [1].Therefore, prompt diagnosis and treatment are necessary. Traditionally, surgical incision and drainage (I\&D) was the mainstay of treatment for DNIs [1,2]; however, surgical and anesthetic procedures may cause adverse events, and surgical approaches in infections of parapharyngeal and retropharyngeal spaces are more difficult to perform than in other deep neck spaces.

Kawasaki disease (KD), the most common cause of acquired heart disease in children in developed countries, is a systemic vasculitis diagnosed based on clinical manifestations including fever lasting for $\geq 5$ days and four of five clinical findings: extremity changes, rash, conjunctivitis, oral changes, and cervical lymphadenopathy (LAP) [3]. Because the incidence of KD is higher in East Asian children than in Western children [3], KD should be considered for the differential diagnosis in East Asian children with prolonged fever. However, KD can manifest as

Copyright (C) 2020 by Korean Society of Otorhinolaryngology-Head and Neck Surgery.

This is an open-access article distributed under the terms of the Creative Commons Attribution Non-Commercial License (https://creativecommons.org/licenses/by-nc/4.0)

which permits unrestricted non-commercial use, distribution, and reproduction in any medium, provided the original work is properly cited. 
only fever and cervical LAP or accompanying retropharyngeal inflammation [4-8]. Although infectious cervical LAP and DNI should be excluded in these patients due to their different treatment strategies, comparative studies between children with KD and accompanying retropharyngeal inflammation and those with DNIs are rare [4-6], compared to studies comparing children with KD and accompanying cervical LAP and those with infectious cervical LAP [7-11].

At our hospital, we experienced several children who initially presented with DNIs and were eventually diagnosed with KD. This study was performed to evaluate clinical features and outcomes of Korean children initially diagnosed with parapharyngeal and retropharyngeal inflammation (cellulitis and abscess) using a neck computed tomography (CT).

\section{MATERIALS AND METHODS}

\section{Subject}

Children in whom a neck CT was performed for suspicious DNI were recruited from among children admitted to the Department of Pediatrics, Seoul St. Mary's Hospital, Seoul, Korea between January 2013 and December 2017. Children with cellulitis or abscess in the parapharyngeal or retropharyngeal space based on neck CT findings were included in the present study. Immunocompromised children, traumatized children, and those with cervical structural anomalies were excluded. Children with a neck CT showing peritonsillar abscess, superficial cellulitis, or superficial lymph node abscess without accompanying parapharyngeal or retropharyngeal inflammation were also excluded. This study was approved by the Institutional Review Board of Seoul St. Mary's Hospital with a waiver of acquiring informed consent (IRB No. KC18RESI0760).

\section{Data collection}

Medical records of the enrolled children were retrospectively reviewed. Demographic data, including sex and age, were collected. Clinical data, including final diagnosis at hospital discharge, fever duration before and after admission, accompanying symptoms and head and neck examination findings on admission,

\section{HIIGHLIIGHTS}

- A quarter of children presenting with deep neck inflammation were eventually diagnosed with Kawasaki disease.

- Significantly more children with Kawasaki disease were febrile for $\geq 3$ days after admission compared to those with deep neck infections $(P=0.009)$.

- Deep neck abscesses were observed in $44.4 \%$ of children with deep neck infections and in no child with Kawasaki disease $(P=0.009)$. and treatment modality were investigated. White blood cell count, hemoglobin level, platelet count, erythrocyte sedimentation rate, $\mathrm{C}$-reactive protein (CRP) and lactate dehydrogenase levels, and aspartate transaminase (AST) and alanine transaminase (ALT) levels were collected. The location (parapharyngeal or retropharyngeal space) and type (cellulitis or abscess) of deep neck inflammation were confirmed based on neck CT findings. Cellulitis was defined as the presence of non-enhancing hypodense lesions in the parapharyngeal or retropharyngeal space, and abscess was defined as the presence of a hypodense lesion with complete rim enhancement.

\section{Statistical analysis}

The enrolled children were divided into two groups based on final diagnosis at hospital discharge: DNI and KD groups. Demographic, clinical, and laboratory data were compared between established groups. Categorical and continuous factors were compared using Fisher's exact and Mann-Whitney tests, respectively. The SPSS ver. 21.0 (IBM Corp., Armonk, NY, USA) was used for statistical analyses, and statistical significance was defined as $P<0.05$.

\section{RESULTS}

Neck CTs were performed in 96 children during the study period. Among them, 47 children were enrolled in the present study; excluding 49 children without definite parapharyngeal or retropharyngeal inflammation on CT. The median age of the enrolled children was 4 years (range, 0 to 11 years), and 30 (63.8\%) of them were males. Deep neck abscess and cellulitis were identified based on CT findings in $16(34.0 \%)$ and $40(85.1 \%)$ children, respectively. Nine children (19.1\%) had both a deep neck abscess and cellulitis (Table 1$)$. Four $(25.0 \%)$ of the 16 children with a deep neck abscess had concurrent parapharyngeal and retropharyngeal abscesses. Nine $(22.5 \%)$ of 40 children with retropharyngeal cellulitis and one $(6.3 \%)$ of 16 children with parapharyngeal or retropharyngeal abscess had an accompanying peritonsillar abscess. Superficial lymph node abscess was seen in two $(5.0 \%)$ of 40 children with retropharyngeal cellulitis.

\section{Comparison between the $\mathrm{DNI}$ and $\mathrm{KD}$ groups}

The final diagnosis was KD in 11 children (23.4\%) and DNI in 36 children $(76.6 \%)$. The children diagnosed with KD com-

Table 1. Type and location of deep neck inflammation

\begin{tabular}{lc}
\hline Factor & Number \\
\hline Parapharyngeal abscess+retropharyngeal abscess & 4 \\
Parapharyngeal abscess+retropharyngeal cellulitis & 9 \\
Retropharyngeal abscess & 3 \\
Parapharyngeal cellulitis+retropharyngeal cellulitis & 5 \\
Retropharyngeal cellulitis & 26 \\
\hline
\end{tabular}


plained of fever lasting for a median of 2 days (range, 0 to 5 days) on admission, and fever continued for a median of 4 days (range, 2 to 6 days) after admission. Clinical manifestations fulfilling KD diagnostic criteria developed within a median of 6 days (range, 4 to 9 days) of fever in all children with KD. Sex, age, symptoms, and laboratory results on admission were not significantly different between the DNI and KD groups (Table 2). On physical examination, cervical LAP was found in more chil- dren in the KD group than those in the DNI group $(100.0 \%$ vs. $66.7 \%, P=0.044)$. Although the fever duration on admission was not significantly different between the two groups, the fever duration after admission was significantly longer in the KD group than in the DNI group; significantly more children in the KD group experienced fever lasting $\geq 3$ days after admission compared to those in the DNI group $(P=0.009)$. Neck CT revealed a deep neck abscess in $44.4 \%$ of children in the DNI

Table 2. Comparison between children in the DNI and KD groups

\begin{tabular}{|c|c|c|c|}
\hline Factor & DNI group $(n=36)$ & $\operatorname{KD}$ group $(n=11)$ & $P$-value \\
\hline Male sex & $21(58.3)$ & $9(81.8)$ & 0.282 \\
\hline Age (yr) & $4(0-11)$ & $4(0-6)$ & 0.683 \\
\hline \multicolumn{4}{|l|}{ Symptom } \\
\hline Fever & $32(88.9)$ & $11(100.0)$ & 0.560 \\
\hline Cough & $9(25.0)$ & $2(18.2)$ & 1.000 \\
\hline Rhinorrhea & $10(27.8)$ & $2(18.2)$ & 0.703 \\
\hline Sputum & $4(11.1)$ & $1(9.1)$ & 1.000 \\
\hline Sore throat & $15(41.7)$ & $3(27.3)$ & 0.492 \\
\hline Neck pain & $16(44.4)$ & $4(36.4)$ & 0.737 \\
\hline Limited neck motion & $17(47.2)$ & $5(45.5)$ & 1.000 \\
\hline Trismus & $3(8.3)$ & $1(9.1)$ & 1.000 \\
\hline Dysphagia & $9(25.0)$ & $1(9.1)$ & 0.413 \\
\hline \multicolumn{4}{|l|}{ Sign } \\
\hline Pharyngeal injection & $15(41.7)$ & $5(45.5)$ & 1.000 \\
\hline Tonsillar injection & $10(27.8)$ & $5(45.5)$ & 0.292 \\
\hline Palpable cervical lymphadenopathy & $24(66.7)$ & $11(100.0)$ & 0.044 \\
\hline Tonsillar deviation & $2(5.6)$ & 0 & 1.000 \\
\hline \multicolumn{4}{|l|}{ Fever duration (day) } \\
\hline Before admission & $2(0-5)$ & $2(0-5)$ & 0.719 \\
\hline After admission & $2(0-5)$ & $4(2-6)$ & $<0.001$ \\
\hline As a whole & $5(0-7)$ & $6(3-9)$ & $<0.001$ \\
\hline Fever duration after admission (day) & & & 0.009 \\
\hline$<3$ & $27(75.0)$ & $3(27.3)$ & \\
\hline$\geq 3$ & $9(25.0)$ & $8(72.7)$ & \\
\hline Computed tomography finding & & & 0.009 \\
\hline Cellulitis & $29(80.6)$ & $11(100.0)$ & \\
\hline Parapharyngeal cellulitis & $4(11.1)$ & $1(9.1)$ & \\
\hline Retropharyngeal cellulitis & $29(80.6)$ & $11(100.0)$ & \\
\hline Abscess & $16(44.4)$ & 0 & \\
\hline Parapharyngeal abscess & $13(36.1)$ & 0 & \\
\hline Retropharyngeal abscess & $7(19.4)$ & 0 & \\
\hline White blood cell count (cells/mm³) & $16,940(5,850-42,530)$ & $15,680(4,590-22,750)$ & 0.123 \\
\hline Absolute neutrophil count & $12,504(410-36,236)$ & 12,632 (193-19,929) & 0.511 \\
\hline Absolute monocyte count & $1,323(501-3,252)$ & $1,067(409-2,078)$ & 0.236 \\
\hline Hemoglobin (g/dL) & $11.8(10.4-14.1)$ & $11.8(10.8-12.5)$ & 0.326 \\
\hline Platelet count (cells/mm³) & $342,500(179,000-619,000)$ & $308,000(188,000-594,000)$ & 0.302 \\
\hline Aspartate transaminase (IU/L) & $26(16-52)$ & $26(23-80)$ & 0.190 \\
\hline Alanine transaminase (IU/L) & $14(7-46)$ & $13(9-69)$ & 0.543 \\
\hline Lactate dehydrogenase $(\mathrm{IU} / \mathrm{L})^{\mathrm{a})}$ & $538(369-793)$ & $538(448-877)$ & 0.576 \\
\hline Erythrocyte sedimentation rate $(\mathrm{mm} / \mathrm{hr})$ & $60(2-120)$ & $67(14-105)$ & 0.979 \\
\hline C-reactive protein (mg/dL) & $9.68(0.02-21.76)$ & $9.73(0.82-19.88)$ & 0.445 \\
\hline
\end{tabular}

Values are presented as number (\%) or median (range).

$\mathrm{DNI}$, deep neck infection; KD, Kawasaki disease.

a) One child in the DNI group was not tested for lactate dehydrogenase. 

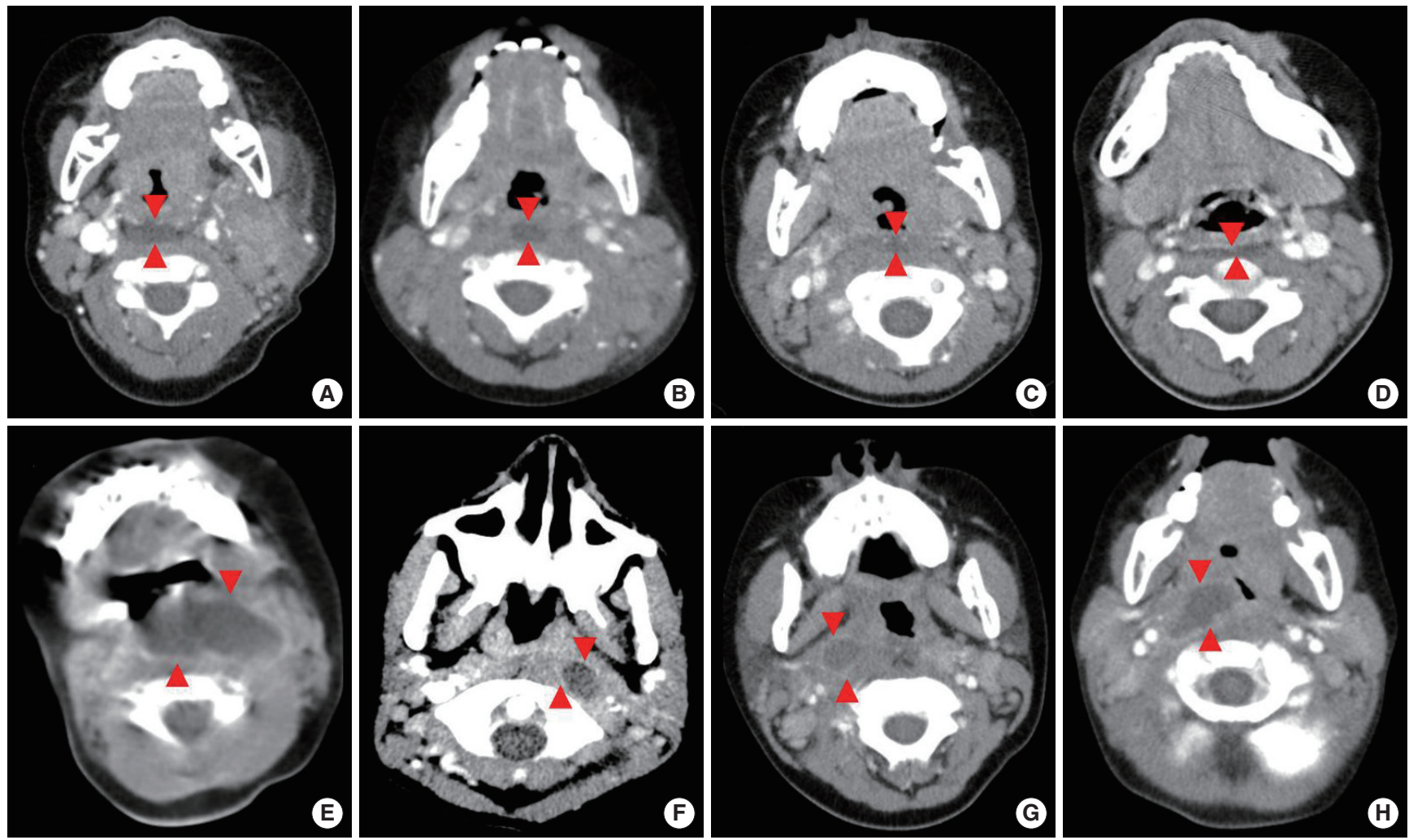

Fig. 1. Neck computed tomography findings of the enrolled children. (A, B) Retropharyngeal cellulitis (arrowheads) in children with Kawasaki disease. (C, D) Retropharyngeal cellulitis (arrowheads) in children with deep neck infection. (E, F) Retropharyngeal abscess with rim enhancement (arrowheads) in children with deep neck infection. (G, H) Parapharyngeal abscess with rim enhancement (arrowheads) in children with deep neck infection.

group and no child in the KD group $(P=0.009)$ (Fig. 1). Among 31 children with deep neck cellulitis without abscess, 11 (35.5\%) experienced fever lasting $\geq 3$ days, and eight $(72.7 \%)$ of them were finally diagnosed with $\mathrm{KD}$ (Fig. 1).

\section{Treatment and outcomes of the DNI group}

Thirty (83.3\%) of the 36 children with DNI were cured with antibiotic therapy alone. The combination of intravenous amoxicillin/clavulanate and clindamycin was the most frequently administered empirical antibiotic regimen (36.1\%) (Table 3). Seven children $(19.4 \%)$ empirically received second- or third-generation cephalosporin and clindamycin combination therapy, and therefore, clindamycin was empirically administered to $55.6 \%$ of the DNI group (Table 3). Eight $(22.2 \%)$ and six (16.7\%) children empirically received amoxicillin/clavulanate and a secondor third-generation cephalosporin, respectively. The remaining two children (5.6\%) empirically received amoxicillin/clavulanate, cefotaxime and clindamycin combination therapy. Glycopeptides were not administered at all. Although fever duration after empirical antibiotic therapy was not significantly different according to empirical antibiotic agents, six $(75.0 \%)$ of the eight children receiving empirical amoxicillin/clavulanate and five (83.3\%) of six children receiving empirical second- or third-generation ceph-
Table 3. Empirical antibiotics administered to children with deep neck infection

\begin{tabular}{lc}
\hline Antibiotics & No. (\%) \\
\hline$\beta$-Lactam agent and clindamycin combination & $22(61.1)$ \\
Amoxicillin/clavulanate+clindamycin & $13(36.1)$ \\
2nd or 3rd generation cephalosporin+clindamycin & $7(19.4)$ \\
Amoxicillin/clavulanate+3rd generation & $2(5.6)$ \\
$\quad$ cephalosporin+clindamycin & \\
$\beta$-Lactam agent & $14(38.9)$ \\
Amoxicillin/clavulanate & $8(22.2)$ \\
2nd or 3rd generation cephalosporin & $6(16.7)$ \\
\hline
\end{tabular}

alosporin eventually received clindamycin add-on therapy based on clinical response.

Six children $(16.7 \%)$ in the DNI group underwent surgical intervention: aspiration in two children and I\&D in four children. Surgical intervention was performed to treat deep neck abscess in three $(50.0 \%)$ children; whereas, the remaining three children $(50.0 \%)$ underwent surgical treatment for accompanying peritonsillar or superficial lymph node abscess. Aspiration for parapharyngeal and retropharyngeal abscesses was performed in one child due to prolonged fever until the 6th day of admission, and I\&Ds were performed for a retropharyngeal abscess in one 
child due to respiratory distress that developed after admission and a parapharyngeal abscess in another child due to an undefined cause based on the attending physician's decision. The longest diameters of abscesses in the three children who underwent surgical intervention were $8.0 \mathrm{~mm}, 30.7 \mathrm{~mm}$, and $41.0 \mathrm{~mm}$, respectively. The median of longest abscess diameter in the 13 children not undergoing surgical intervention was $22.0 \mathrm{~mm}$ (range, 10 to $40 \mathrm{~mm}$ ). Among the three patients who underwent surgical treatment despite a diagnosis of deep neck cellulitis, aspiration was performed for a peritonsillar abscess in one child due to respiratory distress on admission, and I\&Ds were performed for superficial lymph node abscesses in the remaining two children due to impending rupture.

\section{Treatment and outcomes of the KD group}

All of the 11 children diagnosed with $\mathrm{KD}$ received intravenous immunoglobulin (IVIG, $2 \mathrm{~g} / \mathrm{kg}$ ). One of them received a second dose of IVIG therapy due to relapsing fever 3 days after the first dose. Their fever disappeared within 24 hours and 3 days after IVIG administration in six $(54.5 \%)$ and the remaining five children $(45.5 \%)$, respectively. Coronary complications on echocardiography were identified in two $(18.2 \%)$ of the KD patients initially, which resolved after 2 months of aspirin therapy in one and leaved coronary aneurysm in the other patient.

\section{DISCUSSION}

In the present study, a quarter of children initially diagnosed with DNIs based on neck CT findings were eventually diagnosed with KD. Deep neck abscesses were observed more frequently in children with DNIs than in those with $\mathrm{KD}$; however, the fever duration after admission was significantly longer in children with KD than in those with DNIs.

Retropharyngeal inflammation was observed in $3.6 \%$ of patients with KD [4]; however, the exact incidence of retropharyngeal inflammation in patients with $\mathrm{KD}$ has not been defined because cervical imaging studies are not a routine examination for diagnosing KD. Because the incidence of KD is higher in East Asian children including Koreans than in Western children [3,12] and KD can accompany deep neck inflammation [4-6], clinicians should be suspicious for KD in East Asia children presenting with fever and cervical LAP with accompanying symptoms and signs of DNI, such as neck pain and swelling, odynophagia, dysphagia, trismus, neck mass, and limited neck motion [1]. Early differentiation between KD with deep neck inflammation and DNI should be necessary for an appropriate treatment. In previous studies, children with KD who initially presented with only fever and cervical LAP showed high CRP, AST, and ALT levels and higher frequency of retropharyngeal inflammation on neck CT compared to children with infectious cervical LAP [711]. Most of clinical and laboratory findings were not signifi- cantly different between children with KD and those with retropharyngeal infection, with the exception of a higher frequency of neck pain and dysphagia in children with retropharyngeal infection compared to those with KD [4]. The present study showed no significant differences in clinical and laboratory factors on admission between children with KD and those with DNI. However, after admission, fever subsided within 3 days of antibiotic therapy in most children with DNI, while fever persisted for 3 days or longer in most children with $\mathrm{KD}$ despite antibiotic therapy. In a previous report, fever lasted longer after admission in children with KD presenting with only fever and cervical LAP than in those with infectious cervical LAP [7]. In addition to persistent fever, clinical features further suggesting KD developed within 5 to 7 days of hospitalization in almost all patients who were eventually diagnosed with KD both in previous reports and the present study $[4,5,7,9,13]$. Neck CT revealed an abscess in none of the children with $\mathrm{KD}$ in the present study, and previous studies also reported cellulitis rather than abscess in most children diagnosed with $\mathrm{KD}[5,7,8]$. Therefore, KD should be considered when fever persists for more than 3 days despite antibiotic therapy in children with CT-identified deep neck cellulitis. These children should be closely monitored for additional manifestations of KD.

Traditionally, surgical intervention was the mainstay of treatment for DNIs [2,14]. However, parapharyngeal and retropharyngeal spaces are more difficult to access surgically than other deep neck spaces, such as the peritonsillar and submandibular spaces, and adverse events arising from surgical and anesthetic procedures should be considered. Many researchers have reported successful antibiotic therapy without surgical intervention in children with DNIs [15-21]. Surgical intervention was performed in $16.7 \%$ of children with DNIs in the present study; $8.3 \%$ when three children undergoing surgical intervention for accompanying peritonsillar and superficial lymph node abscesses were excluded. In the present study, children with DNIs became afebrile after a median of 2 days of antibiotic therapy; leaving $8.3 \%$ of children febrile after 4 days of antibiotic therapy. Children with KD, who never need surgical intervention, showed clinical features fulfilling KD diagnostic criteria after a median of 3 days of hospitalization. Therefore, empirical antibiotic therapy for 3 to 4 days after admission rather than early surgical intervention can be appropriate in children with deep neck inflammation. Surgical intervention can be considered in children with fever persisting after 3 days of antibiotic therapy after KD has been excluded if they do not present with lifethreatening complications including respiratory distress.

The present study has some limitations including biases arising from its retrospective design. First, the number of enrolled children was small. We excluded children with inflammation of superficial lymph nodes, and peritonsillar, submandibular, and parotid spaces because the decision to proceed with surgical intervention can be made more easily in these patients compared 
to those with parapharyngeal and retropharyngeal inflammation. Second, the DNI group may have included patients with cervical LAP not caused by infection because a specimen to identify infectious microorganisms was not collected from most of the enrolled children. However, the favorable outcomes of enrolled children after antibiotic therapy suggest infectious causes.

In conclusion, a portion of Korean children presenting with deep neck inflammation were eventually diagnosed with KD. Therefore, KD should be considered in children showing deep neck inflammation unresponsive to empirical antibiotic therapy after 3 days, especially in children presenting with deep neck cellulitis rather than deep neck abscess.

\section{CONFLICT OF INTEREST}

No potential conflict of interest relevant to this article was reported.

\section{ORCID}

$\begin{array}{ll}\text { Sooyeon Lim } & \text { https://orcid.org/0000-0002-8929-3377 } \\ \text { Na Young Lee } & \text { https://orcid.org/0000-0002-2402-9321 } \\ \text { Seung Beom Han } & \text { https://orcid.org/0000-0002-1299-2137 } \\ \text { Dae Chul Jeong } & \text { https://orcid.org/0000-0003-0934-817X } \\ \text { Jin Han Kang } & \text { https://orcid.org/0000-0003-1610-6742 }\end{array}$

\section{AUTHOR CONTRIBUTIONS}

Conceptualization: SBH, JHK. Data curation: SL, NYL. Formal analysis: SBH, DCJ. Methodology: SBH, JHK. Writing - original draft: SBH, SL, NYL.Writing - review \& editing: DCJ, JHK.

\section{REFERENCES}

1. Vieira F, Allen SM, Stocks RM, Thompson JW. Deep neck infection. Otolaryngol Clin North Am. 2008 Jun;41(3):459-83.

2. Kirse DJ, Roberson DW. Surgical management of retropharyngeal space infections in children. Laryngoscope. 2001 Aug;111(8):141322.

3. McCrindle BW, Rowley AH, Newburger JW, Burns JC, Bolger AF, Gewitz M, et al. Diagnosis, treatment, and long-term management of Kawasaki disease: a scientific statement for health professionals from the American Heart Association. Circulation. 2017 Apr;135(17): e927-99.

4. Tona R, Shinohara S, Fujiwara K, Kikuchi M, Kanazawa Y, Kishimo- to I, et al. Risk factors for retropharyngeal cellulitis in Kawasaki disease. Auris Nasus Larynx. 2014 Oct;41(5):455-8.

5. Nomura O, Hashimoto N, Ishiguro A, Miyasaka M, Nosaka S, Oana S, et al. Comparison of patients with Kawasaki disease with retropharyngeal edema and patients with retropharyngeal abscess. Eur J Pediatr. 2014 Mar;173(3):381-6.

6. Roh K, Lee SW, Yoo J. CT analysis of retropharyngeal abnormality in Kawasaki disease. Korean J Radiol. 2011 Nov-Dec;12(6):700-7.

7. Kanegaye JT, Van Cott E, Tremoulet AH, Salgado A, Shimizu C, Kruk P, et al. Lymph-node-first presentation of Kawasaki disease compared with bacterial cervical adenitis and typical Kawasaki disease. J Pediatr. 2013 Jun;162(6):1259-63.

8. Park BS, Bang MH, Kim SH. Imaging and clinical data distinguish lymphadenopathy-first-presenting Kawasaki disease from bacterial cervical lymphadenitis. J Cardiovasc Imaging. 2018 Dec;26(4):23846.

9. Yanagi S, Nomura Y, Masuda K, Koriyama C, Sameshima K, EguchiT, et al. Early diagnosis of Kawasaki disease in patients with cervical lymphadenopathy. Pediatr Int. 2008 Apr;50(2):179-83.

10. Katsumata N, Aoki J,Tashiro M,Taketomi-Takahashi A, Tsushima Y. Characteristics of cervical computed tomography findings in kawasaki disease: a single-center experience. J Comput Assist Tomogr. 2013 Sep-Oct;37(5):681-5.

11. Nozaki T, MoritaY, Hasegawa D, Makidono A, YoshimotoY, Starkey J, et al. Cervical ultrasound and computed tomography of Kawasaki disease: comparison with lymphadenitis. Pediatr Int. 2016 Nov; 58(11):1146-52.

12. Kim GB, Park S, Eun LY, Han JW, Lee SY, Yoon KL, et al. Epidemiology and clinical features of Kawasaki disease in South Korea, 20122014. Pediatr Infect Dis J. 2017 May;36(5):482-5.

13. Jun WY, Ann YK, Kim JY, Son JS, Kim SJ, Yang HS, et al. Kawasaki disease with fever and cervical lymphadenopathy as the sole initial presentation. Korean Circ J. 2017 Jan;47(1):107-14.

14. Page NC, Bauer EM, Lieu JE. Clinical features and treatment of retropharyngeal abscess in children. Otolaryngol Head Neck Surg. 2008 Mar;138(3):300-6.

15. Meyer AC, Kimbrough TG, Finkelstein M, Sidman JD. Symptom duration and CT findings in pediatric deep neck infection. Otolaryngol Head Neck Surg. 2009 Feb;140(2):183-6.

16. Cheng J, Elden L. Children with deep space neck infections: our experience with 178 children. Otolaryngol Head Neck Surg. 2013 Jun; 148(6):1037-42.

17. Hoffmann C, Pierrot S, Contencin P, Morisseau-Durand MP, Manach Y, Couloigner V. Retropharyngeal infections in children. Treatment strategies and outcomes. Int J Pediatr Otorhinolaryngol. 2011 Sep;75(9):1099-103.

18. Wong DK, Brown C, Mills N, Spielmann P, Neeff M. To drain or not to drain: management of pediatric deep neck abscesses: a case-control study. Int J Pediatr Otorhinolaryngol. 2012 Dec;76(12):1810-3.

19. Nagy M, Pizzuto M, Backstrom J, Brodsky L. Deep neck infections in children: a new approach to diagnosis and treatment. Laryngoscope. 1997 Dec;107(12 Pt 1):1627-34.

20. McClay JE, Murray AD, Booth T. Intravenous antibiotic therapy for deep neck abscesses defined by computed tomography. Arch Otolaryngol Head Neck Surg. 2003 Nov;129(11):1207-12.

21. Daya H, Lo S, Papsin BC, Zachariasova A, Murray H, Pirie J, et al. Retropharyngeal and parapharyngeal infections in children: the Toronto experience. Int J Pediatr Otorhinolaryngol. 2005 Jan;69(1): 81-6. 